\title{
An Algorithm to Reduce the Number of Nodes in Active Spectrum Sensing Via Cooperative Sequential Detection
}

\author{
Tran Thanh Truc $\cdot$ Hyung Yun Kong
}

\begin{abstract}
In this paper, we propose an algorithm to conserve resources of the common control channel in a cognitive radio network by rejecting the redundant users using cooperative spectrum sensing. The proposed scheme is investigated under the paradigm of active spectrum sensing and a sequential detection technique. The algorithm is based on the J-divergence between the hypotheses of non primary user operation and the otherwise case. We select the most significant eigenvalues, which primarily affect the global statistical test. For the case where interference is from a secondary system transmission, a match filter is first employed to remove the degradation, and then the proposed algorithm is employed to remove the cooperative sensing nodes. Numerical results are provided and compared with conventional cases in order to validate the performance of the proposed algorithm.
\end{abstract}

Key words: Cognitive Radio, Spectrum Sensing, Sequential Detection.

\section{Introduction}

The continuous increase in wireless services and the accompanying great increment in radio spectrum demand are now revealing the scarcity of any new radio frequency band allocation. This, in turn, reveals the need for a secondary system that is able to coexist with existing licensed spectra. Fortunately, the scarcity of radio spectra is actually related to the ineffective utilization of the currently granted spectrum rather than representing a real shortage. A survey of the fixed granted band ranging from $30 \mathrm{MHz}$ to $3 \mathrm{GHz}$ has indicated the presence of numerous white spaces that would be available for a secondary system [1]. The Federal Communication Commission (FCC) has shown interest in developing and investigating a secondary system for the white spaces in TV bands [2].

Any secondary system has to be able to guarantee the first priority of the primary one. The new system also has to have a low cost for infrastructure installation and must be compatible with the legacy primary system. In this sense, because of its ability to sense the presence of licensed users, Cognitive Radio (CR) is a competitive candidate compared to others such as data base registry or beacon signaling approaches [1].
A number of studies have proposed cooperative spectrum sensing models that rely on multi-radio observation to overcome the obstacles of the channel, noise uncertainty, and long sensing period [3] [7]. These models fall into two categories: concurrent combination of the cooperative sensing results [3] [5] and sequential combination of the reported sensing results [6], [7]. Recently, a particular sequential detection paradigm was proposed by Qiyue Zou, et al. [8], which provides a robust approach to obtain a quick detection for spectrum sensing. The scheme allows each cooperative node to forward each observation sample to the base station (BS) for global sensing. The proposal also considers the uncertainty of noise in the sensing operation.

Cooperative sensing models have been quite beneficial for resolving the limitations of local spectrum sensing. However, cooperative sensing is still limited in the periodically quiet sensing time when neither transmission nor reception by the secondary user is allowed. A recent paper [9] has introduced a scheme called active spectrum sensing, where the transmission or reception intervals conventionally prohibited from any sensing operation can be exploited for spectrum sensing. In this paradigm, the users that are free from both signal transmission and reception (we refer to this type of user as

Manuscript received November 24, 2011 ; Revised January 17, 2012 ; Accepted February 27, 2012. (ID No. 20111124-036J)

Dept. of Electrical. Engineering, University of Ulsan, Ulsan, Korea.

Corresponding Author : Hyung Yun Kong (e-mail : hkong@mail.ulsan.ac.kr)

This is an Open-Access article distributed under the terms of the Creative Commons Attribution Non-Commercial License (http://creativecommons.org/licenses/ by-nc/3.0) which permits unrestricted non-commercial use, distribution, and reproduction in any medium, provided the original work is properly cited. 
a free secondary user in the scope of the paper) are permitted to use the active period for sensing. This has extended the ability of local sensing since a larger number of samples are employed to carry out spectrum detection.

Although the reliability of local sensing is much improved, the geographical discrimination among secondary users (SUs) can result in large differences in their observations. For example, due to their geographically separate positions, some users will sense the on active state of primary user (PU), while the others have the opposite result. Hence, the cooperative scheme is actually a useful method in this situation. Conservation of power in each device is important, so further reduction of the sample size is still necessary to decrease the on operating state time. The device will be allowed to assume a sleep mode as soon as possible to save power.

In this paper, we expand upon previous work and investigate a model where several free SUs cooperate to implement spectrum detection during their active periods. We also consider the sequential detection as proposed previously [8] in the paradigm of a cooperative model. This cooperative detection scheme provides very quick detection, on the one hand, while the active sensing paradigm, on the other hand, supplies the large limit for the sample size. Thus, the combination of both methods could bring about a robust detection performance. Using this model, we propose algorithms to reduce the number of nodes that participate in the cooperative sensing. We also analyze the degradation caused by interference originating from BS to SU transmission and introduce a method that uses the matched filter to reduce this effect. The structure of the article is divided into 5 parts: (I) Introduction, (II ) System model, (III) Performance analysis, (IV) Numerical simulation, (V) Conclusion.

\section{II . System Model}

In this scheme, we model PU signal $s$ as the Gaussian signal with a zero mean and variance $\sigma_{s}^{2}$, the noise of the user m-th $n_{m}$ is AWGN, $n_{m} \sim N\left(0, \sigma_{m}^{2}\right)$. The secondary transmission signal carried out by the BS to a specific $\mathrm{SU}$ is denoted by $z$ where $z \sim N\left(0, \sigma_{z}^{2}\right)$. For the conventional case in which there is only the spectrum sensing operation, the model can be constructed as the two hypotheses of no PU signal with the received signal at the BS is: $H_{0}: x_{m}=n_{m}$ and having PU signal with: $H_{1}: x_{m}=h_{m} s+n_{m}$ where $h_{m}$ is denoted as the channel gain between the SU source to the $m$-th user. Because the active sensing scheme allows the BS to car- ry out the transmission to a specific SU during the sensing time, the other nearby SUs may experience interference from this signal, for the no PU presence case: $\tilde{H}_{0}: x_{m}=l_{m} z+n_{m}$, or for the PU in active mode: $\tilde{H}_{1}: x_{m}$ $=h_{m} s+l_{m} z+n_{m}$, where $l_{m}$ is channel gain describing the gain to transmit the signal from the BS to $\mathrm{m}$-th user and back from m-th user to BS. $\sigma_{0, m}^{2}, \sigma_{1, m}^{2}, \tilde{\sigma}_{0, m}^{2}, \tilde{\sigma}_{1, m}^{2}$ denote, respectively, the variances of the received signal at $\mathrm{BS}$ for cases of $H_{0}, H_{1}, \tilde{H}_{0}, \tilde{H}_{1}$. We can easily obtain that $\sigma_{0, m}^{2}=\sigma_{m}^{2}, \sigma_{1, m}^{2}=h_{m}^{2} \sigma_{s}^{2}+\sigma_{m}^{2}, \quad \tilde{\sigma}_{0, m}^{2}=l_{m} \sigma_{z}^{2}+\sigma_{m}^{2} \quad$ and $\quad \tilde{\sigma}_{1, m}^{2}=$ $h_{m}^{2} \sigma_{s}^{2}+l_{m}^{2} \sigma_{z}^{2}+\sigma_{m}^{2}$. Under the scheme of sequential detection, as proposed in [8], each user reports its log-likelihood test in a sample-by-sample manner. The statistical test of the $\mathrm{m}$-th user at $\mathrm{n}$-th sample is $\operatorname{LLR}(n, m)=$ $\log p_{1, m}-\log p_{0, m}$ (LLR stands for the $\log$ likelihood ratio test). The combination is undertaken by the BS as follows:

$$
L L R=\sum_{n=1}^{N} \sum_{m=1}^{M} L L R(n, m)
$$

Under the context of no SU transmission, the test is:

$$
L L R=\sum_{n=1}^{N} \sum_{m=1}^{M} \frac{1}{2}\left(\left(\frac{1}{\sigma_{0, m}^{2}}-\frac{1}{\sigma_{1, m}^{2}}\right) x_{m}^{2}[n]+\log \left(\frac{\sigma_{0, m}^{2}}{\sigma_{1, m}^{2}}\right)\right)
$$

\section{Performance Analysis}

\section{3-1 Non SU Interference}

In this sub-section, we investigate the performance of the case when no SU signal is transmitted during the sensing time. Here, $A$ and $B$ are alternatively the threshold for the hypothesis $H_{0}$ or $\tilde{H}_{0}$ and thresholds for $H_{1}$ or $\tilde{H}_{1}$. As introduced in [8], these thresholds do not depend on the distribution of the received signal. The threshold values are as given : $A=\log \left(\frac{1-\beta}{\alpha}\right)$ and $B=\log \left(\frac{1-\alpha}{\beta}\right)$ where $\alpha, \alpha<<1$, is the false alarm probability and $\beta, \beta<<1$, is the missed detection probability. The average samples number (ASN) for the decision ' 0 ', which denotes for no PU presence in the case of $H_{0}$, is given as:

$$
E_{H_{0}}\left\{N_{\text {stop }}\right\}=\frac{-\left(A-B-A e^{B}+B e^{A}\right) /\left(e^{A}-e^{B}\right)}{\left(\sigma_{0, m}^{2} /\left(2 \sigma_{1, m}^{2}\right)\right)-1 / 2+\log \left(\sigma_{1, m}^{2} / \sigma_{0, m}^{2}\right) / 2}
$$

and for the decision ' 1 ' denoting the PU presence, the ASN is expressed as: 


$$
E_{H_{1}}\left\{N_{\text {stop }}\right\}=\frac{\left(A-B-A e^{-B}+B e^{-A}\right) /\left(e^{-A}-e^{-B}\right)}{\left(\sigma_{1, m}^{2} /\left(2 \sigma_{0, m}^{2}\right)\right)-1 / 2-\log \left(\sigma_{1, m}^{2} / \sigma_{0, m}^{2}\right) / 2}
$$

For large ASN, the Central Limit Theorem allows approximation of the LLR distribution to the Gaussian distribution as follows:

$$
\left\{\begin{array}{l}
H_{0}: L L R \sim N\left(\mu_{0}, \sigma_{0}^{2}\right) \\
H_{1}: L L R \sim N\left(\mu_{1}, \sigma_{1}^{2}\right)
\end{array}\right.
$$

with $\mu_{0}=\frac{N_{\text {stop }}}{2} v_{0}, \sigma_{0}^{2}=\frac{N_{\text {stop }}}{2} \rho_{0}^{2}, \mu_{1}=\frac{N_{\text {stop }}}{2} v_{1}, \sigma_{1}^{2}=\frac{N_{\text {stop }}}{2} \rho_{1}^{2}$, where

$$
\begin{aligned}
& v_{0}=\left(\sum_{m=1}^{M}\left(1-\frac{\sigma_{0, m}^{2}}{\sigma_{1, m}^{2}}\right)+\sum_{m=1}^{M} \log \left(\frac{\sigma_{0, m}^{2}}{\sigma_{1, m}^{2}}\right)\right) \\
& \rho_{0}^{2}=\left(\sum_{m=1}^{M}\left(1-\frac{\sigma_{0, m}^{2}}{\sigma_{1, m}^{2}}\right)^{2}\right) \\
& v_{1}=\left(\sum_{m=K+1}^{M}\left(\frac{\sigma_{1, m}^{2}}{\sigma_{0, m}^{2}}-1\right)+\sum_{m=1}^{M} \log \left(\frac{\sigma_{0, m}^{2}}{\sigma_{1, m}^{2}}\right)\right) \\
& \rho_{1}=\left(\frac{\sigma_{1, m}^{2}}{\sigma_{0, m}^{2}}-1\right)^{2}
\end{aligned}
$$

\section{3-2 The Uncorrelated SU Interference}

So far, equation (2) has only considered the case where the received sensing signal is not contaminated by the SU interference. However, due to the active sensing, the BS may transmit its data to a specific user. Due to imperfect beamforming, a neighbor user can be affected by this transmitted SU signal leakage. In response to this interference, the LLR distribution will differ from the LLR-test analysis results. Hence, the LLR equation should be changed to follow the actual LLR distribution, which is given as:

$$
\left\{\begin{array}{l}
\tilde{H}_{0}: L L R \sim N\left(\tilde{\mu}_{0}, \tilde{\sigma}_{0}^{2}\right) \\
\tilde{H}_{1}: L L R \sim N\left(\tilde{\mu}_{1}, \tilde{\sigma}_{1}^{2}\right)
\end{array}\right.
$$

where $\tilde{\mu}_{0}=\frac{N_{\text {stop }}}{2} \tilde{v}_{0}(K), \quad \tilde{\sigma}_{0}^{2}=\frac{N_{\text {stop }}}{2} \tilde{\rho}_{0}^{2}(K), \quad \tilde{\mu}_{1}=\frac{N_{\text {stop }}}{2} \tilde{v}_{1}(K)$, $\tilde{\sigma}_{1}^{2}=\frac{N_{\text {stop }}}{2} \tilde{\rho}_{1}^{2}(K)$ with

$$
\begin{aligned}
& \tilde{v}_{0}(K)=\left(\sum_{m=1}^{K} \tilde{\sigma}_{0, m}^{2}\left(\frac{1}{\sigma_{0, m}^{2}}-\frac{1}{\sigma_{1, m}^{2}}\right)+\sum_{m=K+1}^{M}\left(1-\frac{\sigma_{0, m}^{2}}{\sigma_{1, m}^{2}}\right)+\sum_{m=1}^{M} \log \left(\frac{\sigma_{0, m}^{2}}{\sigma_{1, m}^{2}}\right)\right) \\
& \tilde{\rho}_{0}^{2}(K)=\left(\sum_{m=1}^{K} \tilde{\sigma}_{0, m}^{4}\left(\frac{1}{\sigma_{0, m}^{2}}-\frac{1}{\sigma_{1, m}^{2}}\right)^{2}+\sum_{m=K+1}^{M}\left(1-\frac{\sigma_{0, m}^{2}}{\sigma_{1, m}^{2}}\right)^{2}\right)
\end{aligned}
$$

$$
\begin{aligned}
& \tilde{\nu}_{1}(K)=\left(\sum_{m=1}^{K} \tilde{\sigma}_{1, m}^{2}\left(\frac{1}{\sigma_{0, m}^{2}}-\frac{1}{\sigma_{1, m}^{2}}\right)+\sum_{m=K+1}^{M}\left(\frac{\sigma_{1, m}^{2}}{\sigma_{0, m}^{2}}-1\right)+\sum_{m=1}^{M} \log \left(\frac{\sigma_{0, m}^{2}}{\sigma_{1, m}^{2}}\right)\right) \\
& \tilde{\rho}_{1}^{2}(K)=\left(\sum_{m=1}^{K} \tilde{\sigma}_{1, m}^{4}\left(\frac{1}{\sigma_{0, m}^{2}}-\frac{1}{\sigma_{1, m}^{2}}\right)^{2}+\sum_{m=K+1}^{M}\left(1-\frac{\sigma_{1, m}^{2}}{\sigma_{0, m}^{2}}\right)^{2}\right)
\end{aligned}
$$

$K$ denotes the number of user being affected by the SU signal leakage.

From this situation, since the statistical test does not match with the practice, the threshold $A$ and $B$ cannot hold the false alarm and missed detection probability $P_{f}=\alpha, P_{M}=\beta$ as designed. For a value of the stop ASN $N_{\text {stop }}$, the false alarm and missed detection probability are given as:

$$
\begin{aligned}
& P_{f}=Q\left(\frac{A-\tilde{\mu}_{0}}{\tilde{\sigma}_{0}}\right)=Q\left(\frac{Q^{-1}(\alpha) \sqrt{\frac{N_{A}}{2} \rho_{0}^{2}}-\frac{N_{A}}{2} \nu_{0}-\frac{\tilde{S}_{\text {sop }}}{2} \tilde{\nu}_{0}(K)}{\sqrt{\frac{N_{\text {sop }}}{2}} \tilde{\rho}_{0}(K)}\right) \\
& P_{M}=Q\left(\frac{\tilde{\mu}_{1}-B}{\tilde{\sigma}_{1}}\right)=Q\left(\frac{\left.\frac{\frac{N_{\text {stop }}}{2} \tilde{V}_{1}(K)-Q^{-1}(\beta) \sqrt{\frac{N_{B}}{2} \rho_{1}^{2}}-\frac{N_{B}}{2} v_{1}}{\sqrt{\frac{N_{\text {stop }}}{2}} \tilde{\rho}_{1}(K)}\right)}{\rho_{1}}\right)
\end{aligned}
$$

where $N_{B}=E_{H_{0}}\left\{N_{\text {stop }}\right\}$ and $N_{A}=E_{H_{1}}\left\{N_{\text {stop }}\right\}$, which have been derived in (3) and (4).

\section{3-3 Redundant Node Removal}

\section{3-3-1 Algorithm in the Case of Non SU Interference}

As the number of users increases, more samples are collected to exploit gain sensing. Nevertheless, the limited common control channel restricts the maximum cooperative users. Furthermore, due to collisions among the users when competing for reporting, each log-likelihood sample test can result in increased overall sensing time [7]. In this section, we propose an algorithm to select the most meaningful users that mainly affect the test results, in order to distinguish between two hypotheses. Here, $R_{X, 0}, R_{X, 1}$ denote the covariance matrices of two hypotheses $H_{0}$ and $H_{1}$. For the case of non SU interference, $R_{X, 0}, R_{X, 1}$ can be expressed as:

$$
\begin{aligned}
& R_{X, 0}=\operatorname{diag}\left\{\sigma_{0,1}^{2}, \sigma_{0,2}^{2}, . ., \sigma_{0, m}^{2}\right\} \\
& R_{X, 1}=\operatorname{diag}\left\{\sigma_{1,1}^{2}, \sigma_{1,2}^{2}, . ., \sigma_{1, m}^{2}\right\}
\end{aligned}
$$

The first part of LLR in (2) can be rewritten in the quadratic form as below [9]:

$$
\begin{aligned}
L L R^{\prime} & =X^{T}\left(R_{X, 0}^{-1}-R_{X, 1}^{-1}\right) X \\
& =Y^{T}\left(I-\Lambda^{-1}\right) Y
\end{aligned}
$$


with $X=\left[x_{1}, x_{2}, . ., x_{M}\right]^{T}, \quad Y=\left[y_{1}, y_{2}, . ., y_{M}\right]^{T}, \quad Y=U^{T} R_{X, 0}^{-1 / 2} X$, $R_{X, 0}^{-1 / 2} R_{X, 1} R_{X, 0}^{-T / 2}=U^{T} \Lambda U$ and $I$ is the unit matrix. The matrix $\Lambda$ is the diagonal and contains the eigenvalues of $\lambda_{m}, m=1 . ., M$ the matrix $R_{X, 0}^{-1 / 2} R_{X, 1} R_{X, 0}^{-T / 2}$. The above transformation (from $\mathrm{X}$ to $\mathrm{Y}$ ) introduce the special property: $H_{0}: Y \sim N(0, I)$ and $H_{1}: Y \sim N\left(0, U^{T} \Lambda U\right)$.

The J-divergence between the two hypotheses can measure how the $L L R^{\prime}$ can distinguish the between them under the hypothesis test. The J-divergence is given as [10] :

$$
J=\sum_{m=1}^{M}\left[\lambda_{m}+\lambda_{m}^{-1}-2\right]
$$

We can see from (9) that when the $\lambda_{m}$ is unity, its contribution to $J$ is zero. Hence, the users corresponding to these unit eigenvalues should be removed first. Within a given ASN $N_{0}$, the selection method is simply the removal of the lowest $\left|1-\lambda_{m}\right|$ users. Hence, we propose algorithm 1 to remove the unimportant users based on the requirement of $N_{0}$. Without a loss of generality, we can assume that $\lambda_{1}>\lambda_{2}>. .>1>. .>\lambda_{M}>0$. The selected users are saved in the set $S$.

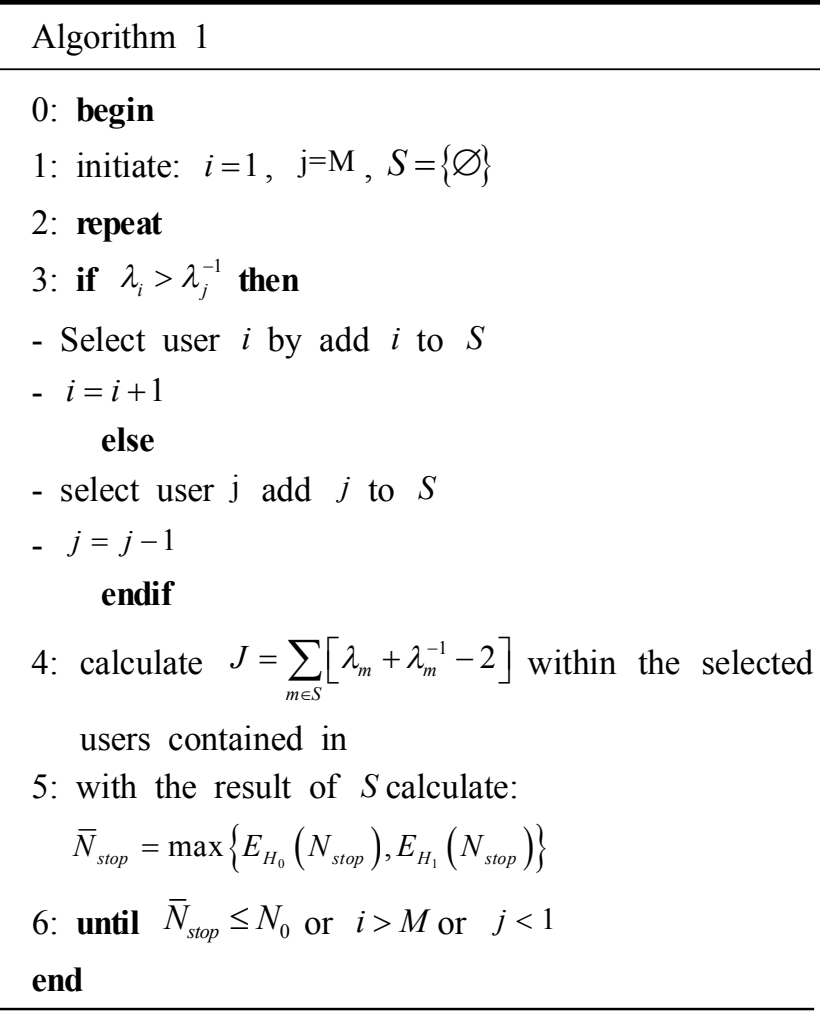

In the case where total J-divergence values of all nodes cannot achieve the requirement of ASN, the algorithm also supplies a method to add all users with ei- genvalues of $\lambda_{m} \neq 0$.

Algorithm 1, however, only considers the covariance matrices of $\mathrm{M}$ users at a single sample. For a general case, the ASN that is equal to $\bar{N}_{\text {stop }}=\max \left\{E_{H_{0}}\left(N_{\text {stop }}\right)\right.$, $\left.E_{H_{1}}\left(N_{\text {stop }}\right)\right\}$ is used to achieve the decision. The covariance matrices $R_{X, 0}, R_{X, 1}$ should therefore be replaced by $\tilde{R}_{X, 0}, \tilde{R}_{X, 1}$ as follows:

$$
\tilde{R}_{X, 0}=\left[\begin{array}{cccc}
R_{X, 0} & 0 & . . & 0 \\
0 & R_{X, 0} & . . & 0 \\
. . & . . & . . & . . \\
0 & 0 & . . & R_{X, 0}
\end{array}\right]_{\left(\bar{N}_{\text {stop }} M\right) \times\left(\bar{N}_{\text {stop }} M\right)}
$$

and

$$
\tilde{R}_{X, 1}=\left[\begin{array}{cccc}
R_{X, 1} & 0 & . . & 0 \\
0 & R_{X, 1} & . . & 0 \\
. . & . . & . . & . . \\
0 & 0 & . . & R_{X, 1}
\end{array}\right]_{\left(\bar{N}_{s t o p} M\right) \times\left(\bar{N}_{\text {stop }} M\right)}
$$

The covariance matrices represent the correlation of the received signal $X=\left[\dot{X}_{1}^{T}, \dot{X}_{2}^{T}, . ., \dot{X}_{N}^{T}\right]^{T}$, with $\dot{X}_{n}=$ $\left[x_{1}(n), . ., x_{M}(n)\right]^{T}$ being the $M$ reported signal at the n-th sample. Similarly, we can employ equation (9) to achieve the eigenvalues $\lambda_{1,1}, . ., \lambda_{1, M} . ., \lambda_{n, m}, . ., \lambda_{n, M}$. Algorithm 1 should be replaced by Algorithm 2 as follows:

Algorithm 2

\section{0 : begin}

1: initiate: $S=\{1,2, . ., M\}$

2: if $\bar{N}_{\text {stop }}=\max \left\{E_{H_{0}}\left(N_{\text {stop }}\right), E_{H_{1}}\left(N_{\text {stop }}\right)\right\}<N_{0}$ then

$/ *$ if all the users are selected and the $\bar{N}_{\text {stop }}$ satisfies the requirement of $N_{0}$ samples*/

3: Calculate J-divergence contributed by each user $J_{m}=\sum_{n=1}^{N}\left[\lambda_{n, m}+\lambda_{n, m}^{-1}-2\right], m=1, . ., M$

4: $\operatorname{Sort}\left(J_{1}, . ., J_{m}, . ., J_{M}\right)$.

* Without loss of the generality, we assuming $J_{1}>. .>J_{m}>. .>J_{M} * /$

5: $j=M$

6: repeat

7: Remove user $j$-th out of $\mathrm{S}$

8: Calculate $\bar{N}_{\text {stop }}$ within the updated $S$

$$
\bar{N}_{\text {stop }}=\max \left\{E_{H_{0}}\left(N_{\text {stop }}\right), E_{H_{1}}\left(N_{\text {stop }}\right)\right\}
$$

9: Calculate $\tilde{R}_{X, 0}, \tilde{R}_{X, 1}, \Lambda$ within the $\bar{N}_{\text {stop }}$ 
10: Calculate J-divergence contributed by each user

11: $J_{m}=\sum_{n=1}^{N}\left[\lambda_{n, m}+\lambda_{n, m}^{-1}-2\right], m=1, . ., M-1$

12: $\operatorname{Sort}\left(J_{1}, . ., J_{m}, . ., J_{j-1}\right)$.

13: Until $\bar{N}_{\text {stop }} \geq N_{0}$

14: Add the last removed user out of $S$

15: end if

16: end

\section{3-3-2 Node Removal for Correlated SU Interference}

In the case where BS starts to transmit data to a specific user, the statistical LLR test pre-designed in (2) does not reflect the true distribution of the received signal. In the scope of this paper, we assume that no delay exists between the SU signal transmitted by the BS and one that contributes to the reported sample $x_{m}(n)$. Thus, the BS can rely on its SU signal transmitted to estimate the power of interference contributing to the received signal, as given in the following:

$$
\hat{P}_{z_{m}}=\left(\int_{0}^{T} x_{m} z d t\right)
$$

From this, the overall channel gain to transmit the SU signal from the BS to the m-th user and back from $\mathrm{m}$-th user to BS is estimated as:

$$
\hat{l}_{m}=\left(\int_{0}^{T} x_{m} z d t\right) / \sigma_{z}^{2}
$$

Relying on this estimation, the BS can calculate $\tilde{\sigma}_{0, m}^{2}$, $\tilde{\sigma}_{1, m}^{2}, R_{X, 0}, R_{X, 1}, \tilde{R}_{X, 0}, \tilde{R}_{X, 1}$ and update the LLR test. In the scheme of SU interference, the received signals contaminated by $z$ are correlated. Hence, the LLR test in (2) is rewritten as follows:

$$
\begin{aligned}
L L R & =\sum_{n=1}^{N_{\text {sop }}}\left(\sum_{m=1}^{K} L L R(n, m)+\sum_{m=K+1}^{M} \operatorname{LLR}(n, m)\right) \\
& =\frac{1}{2} X^{T}\left(R_{X, 0}^{-1}-R_{X, 1}^{-1}\right) X+\frac{1}{2} \log \left(\frac{\left|R_{X, 0}\right|}{\left|R_{X, 1}\right|}\right)
\end{aligned}
$$

After updating the statistical test, the BS is able to apply the proposed node removal algorithm to remove the redundant users to save the control channel resources.

\section{Simulation}

In this part, we survey a case where BS selects the
$M=7$ free SU users in its region where the sensing channel of each has the same value: $h=0.3162$, and the $\mathrm{PU}$ signal variance is assumed at $\sigma_{s}^{2}=1$. Each of $\mathrm{SU}$ users tolerates a number of zero mean AWGN with respective variances: $\sigma_{i}^{2}=1,0.9,0.8,0.7,0.6,0.5,0.4$. The BS to SU signal $z$ is assumed to be a Gaussian signal with a zero mean and unit variance. The channel gain according to $z$ at each user is $l_{m}=.33, .45, .74, .51$, $.39,91, .97$. In this part, we consider two cases of $z$ : the uncorrelated interference between SU users and the correlated interference of each user in terms of this signal.

Fig. 1 illustrates the cases where the statistical test mismatches with the received signal distribution. In this case, the log likelihood test is taken under the case of

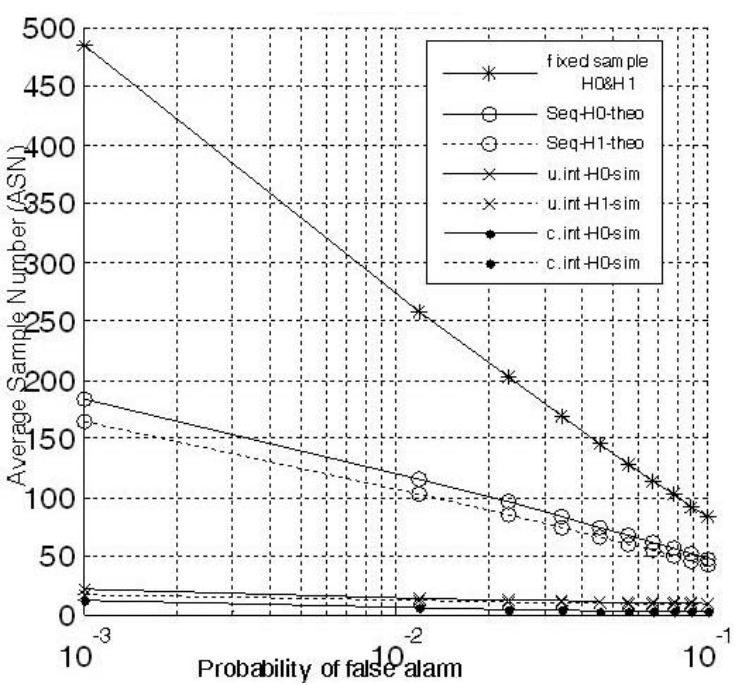

(a) ASN vs PF

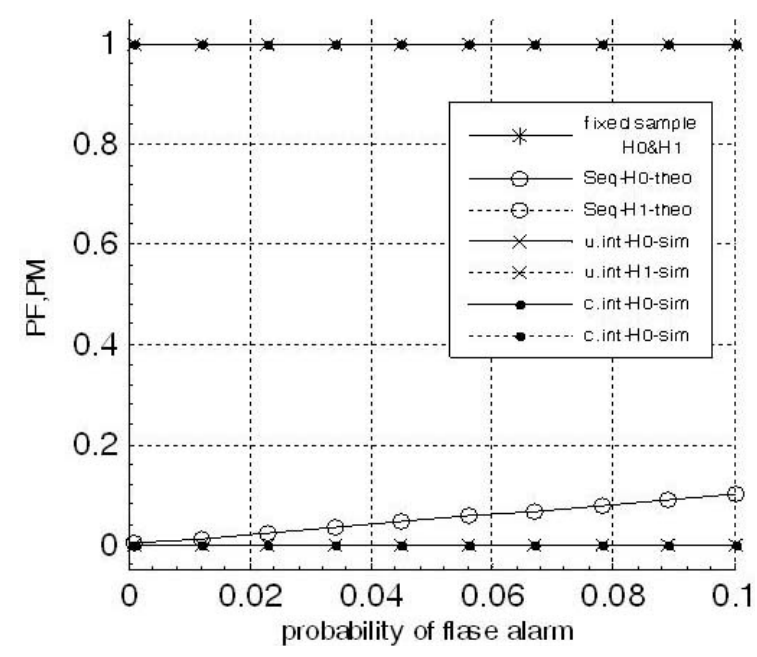

(b) Practical PF, PM vs PF

Fig. 1. Detection performance of using the non-interference statistical test when SUs are in the cases of uncorrelated interference and correlated interference. 


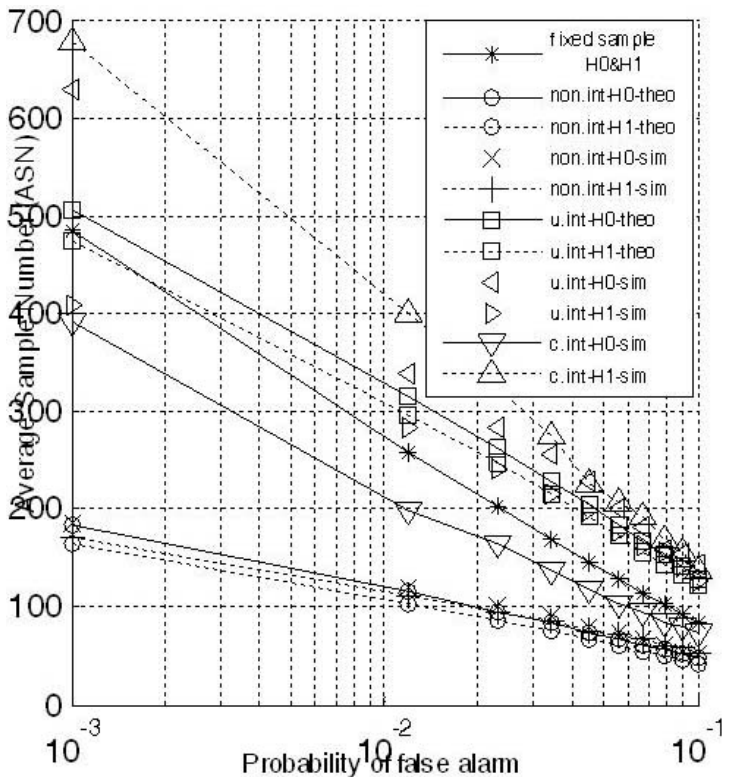

(a) ASN vs PF

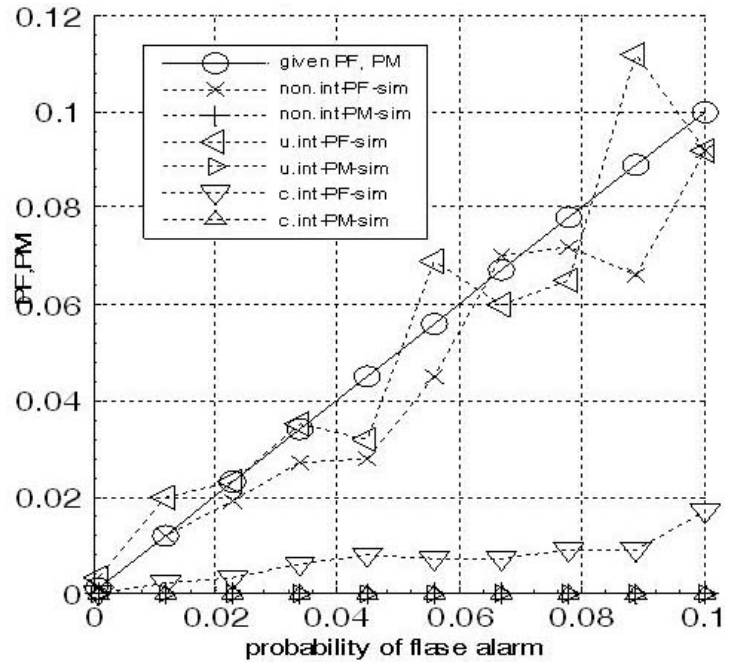

(b) Practical PF, PM vs PF

Fig. 2. Detection performance using suitable statistical tests when SUs are in the three cases of non-interference, uncorrelated interference, and correlated interference.

non-interference at SU. Fig. 1a shows that the ASNs of the received signal contaminated by uncorrelated interference (marked as "u.int" in the figure) and the correlated interference (marked as "c.int) are fairly low in comparison with ASN analyzed by (3) and (4). Nevertheless, Fig. 1(b) indicates that this detection is not correct because the experimental probability of a false alarm is 1 for all cases of the designed false alarm and missed detection probability.

Fig. 2 illustrates the case when the statistical test is used appropriately in each case of non-interference, uncorrelated interference, and correlated interference. The

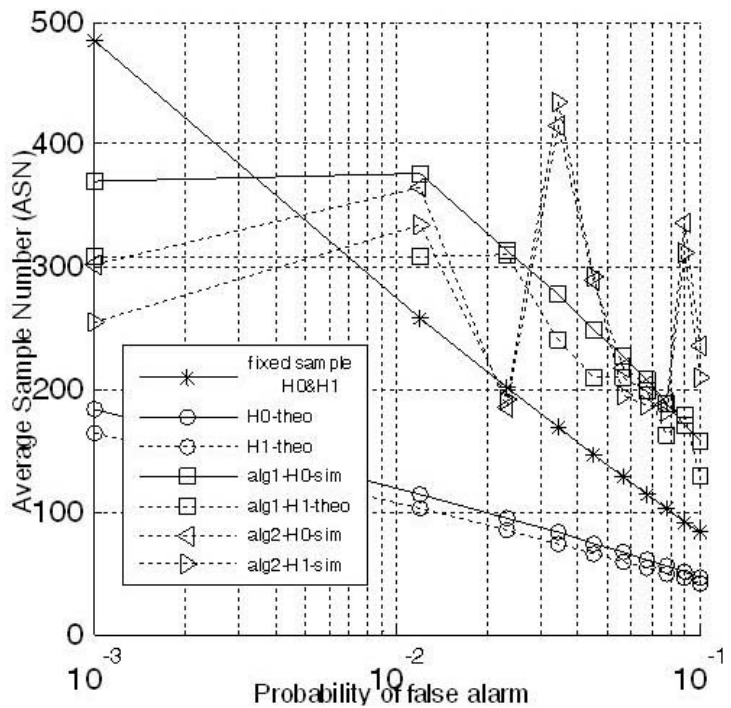

(a) ASN vs PF

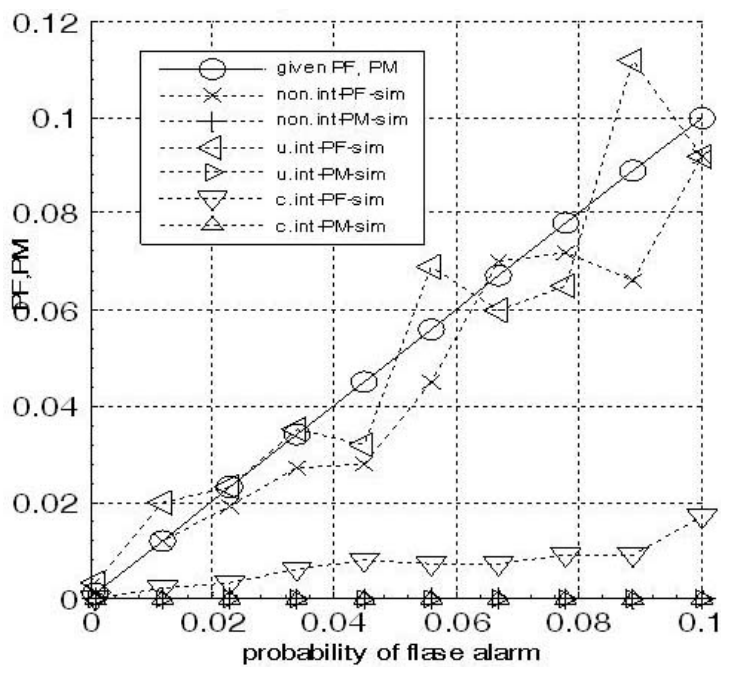

(b) Practical PF, PM vs PF

Fig. 3. The two algorithms for reducing the cooperative node within the constraint of the ASN being below 400 samples.

figure shows that the ASNs for the case of the interference cases are greater than those in Fig. 1. In Fig. 2(b), the false alarm and missed detection probability have reduced to become approximately the designed values.

In Fig. 3, the two algorithms for reducing the number of cooperative nodes are investigated under the non-interference scheme. The figure shows that most of the simulation results lie below the preselected ASN at 400 samples or slightly above this value. Fig 3(b) shows that all of the experimental false alarm and missed detection values are below the preselected maximum value. Hence, this implies that the algorithms have worked correctly.

Fig. 3 describes the performance of the two algorithms in the cases of the non-interference, uncorrelated 
interference, and correlated interference. Fig. 3 shows that the value of the ASNs is less than the required value $N_{0}=500$ and the ASNs do not follow a linear decrease when the false alarm and missed detection increase. Fig. 3(b) shows how many users are selected after the removal. The total number of users has been reduced by almost half.

\section{Conclusion}

In this paper, we considered the scheme of active spectrum sensing in the cooperative model via sequential detection. We have shown that, in the scheme of active spectrum sensing, the uncorrelated interference and the SU transmission signals have been significantly affected by the performance of the sequential detection. Coping with the limited resources in the control channel, we propose two algorithms to reduce the number of cooperative nodes. As the simulations showed, the algorithms are able to eliminate redundant users within the constraint of a preselected ASN while maintaining a detection performance that satisfies system requirements.

\section{References}

[1] A. Ghasemi, E. S. Sousa, "Spectrum sensing in cognitive radio networks: requirements, challenges and design trade-offs," Communications Magazine, IEEE, vol. 46, no. 4, pp. 32-39, 2008.

[2] FCC, "Notice of proposed rulemaking, in the matter of unlicensed operation in the TV broadcast bands (ET Docket no. 04-186) and additional spectrum for unlicensed devices below $900 \mathrm{~s} \mathrm{MHz}$ and in the 3 GHz band (ET Docket no. 02-380)," FCC 04-113, May 2004.

Tran Thanh Truc

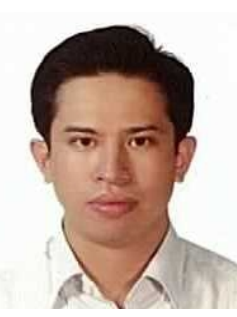

received the B.S degree in Telecommunication Engineering from Poly-technique University of Danang, Vietnam in 2005. In 2008, he got the degree of Master from Hochiminh University of Technology, Vietnam in major of Electrical and Electronics Engineering. Since 2010, he has been studying Ph.D. program at University of Ulsan, Korea. His major research area is Cognitive Radio Network, Cooperative Communication.
[3] M. Matsui, H. Shiba, K. Akabane, and K. Uehara, "A novel cooperative sensing technique for cognitive radio," in Proc. 18th IEEE International Symposium on Personal, Indoor and Mobile Radio Communications (PIMRC'07), pp. 1-5, Sep. 2007.

[4] J. Unnikrishnan, V. V. Veeravalli, "Cooperative sensing for primary detection in cognitive radio," IEEE J. Sel. Topics Signal Process, vol. 2, no. 1, pp. 1827, Feb. 2008.

[5] Z. Quan, S. Cui, and A. H. Sayed, "Optimal linear cooperation for spectrum sensing in cognitive radio networks," IEEE J. Sel. Topics Signal Process, vol. 2, no. 1, pp. 28-40, Feb. 2008.

[6] S. Yeelin, Y. T. Su, "A sequential test based cooperative spectrum sensing scheme for cognitive radios", Personal, Indoor and Mobile Radio Communications (PIMRC) 2008. IEEE 19th International Symposium on, pp. 1-5, 2008.

[7] R. Yu, C. Wei, and C. Zhigang, "A sequential sensing data transmission and fusion approach for large scale cognitive radios", Communications (ICC), 2010 IEEE International Conference on, pp. 1-5, May 2010.

[8] Q. Zou, S. Zheng, and A. Sayed, "Cooperative sensing via sequential detection," Signal Processing, IEEE Transactions on, vol. 58, issue 12, pp. 62666283, 2010.

[9] S. H. Song, K. Hamdi, and K. B. Letaief, "Spectrum sensing with active cognitive systems," Wireless Communications, IEEE Transactions on, vol. 9, no. 6, pp. 1849-1854, Jun. 2010.

[10] L. L. Scharf, Statistical Signal Processing: Detection, Estimation and Time Series Analysis, Addison-Wesley Publishing Company, Inc., 1991.

\section{Hyung Yun Kong}

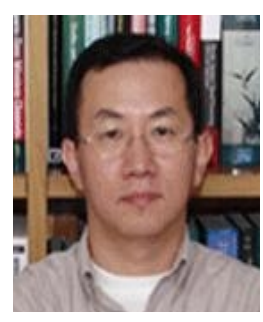

received the M.E. and Ph.D. degrees in electrical engineering from Polytechnic University, Brooklyn, New York, USA, in 1991 and 1996, respectively, He received a $\mathrm{BE}$ in electrical engineering from New York Institute of Technology, New York, in 1989. Since 1996, he has been with LG electronics Co., Ltd., in the multimedia research lab developing PCS mobile phone systems, and from 1997 the LG chairman's office planning future satellite communication systems. Currently he is a Professor in electrical engineering at the University of Ulsan, Korea. His research area includes channel coding, detection and estimation, cooperative communications, cognitive radio and sensor networks. He is a member of IEEK, KICS, KIPS, IEEE, and IEICE. 